\title{
Symmetric Discrete Orthonormal Stockwell Transform
}

\author{
Yanwei Wang* and Jeff Orchard ${ }^{\dagger}$ \\ * Department of Applied Mathematics; \\ ${ }^{\dagger}$ David R. Cheriton School of Computer Science, \\ 200 University Avenue West, \\ University of Waterloo, \\ Waterloo, Ontario, Canada N2L 3G1
}

\begin{abstract}
.
The Stockwell transform (ST) is a time-frequency signal decomposition that is gaining in popularity, likely because of its direct relation with the Fourier Transform (FT). A discrete and non-redundant version of the ST, denoted the Discrete Orthonormal Stockwell Transform (DOST), has made the use of the ST more feasible. However, the matrix multiplication required by the DOST can still be a formidable computation, especially for high-dimensional data. Moreover, the symmetric property of the ST and FT is not present in the DOST.

In this paper, we investigate a new Symmetric Discrete Orthonormal Stockwell Transform (SDOST) that still keeps the non-redundant multiresolution features of the DOST, while maintaining a symmetry property similar to that of the FT.

First, we give a brief introduction for the ST and the DOST. Then we analyze the DOST coefficients and modify the transform to get a symmetric version. A small experiment shows that the SDOST has kept the abilities of the DOST and demonstrates the advantage of symmetry when applying the SDOST.
\end{abstract}

Keywords: Stockwell transform, DOST, symmetric DOST

PACS: PACS Category 00.02.70.Hm

\section{INTRODUCTION}

The Stockwell transform (ST) is a multiresolution transform that represents a time signal in a time-frequency domain, and its coefficients are conjugate symmetric for a real-valued input signal. However, the Discrete Orthonormal Stockwell Transform (DOST) looses the symmetry because of how the time-frequency space is partitioned, making it difficult to manipulate the coefficients for some applications. A non-redundant time-frequency transform that maintains the symmetry property would make the application of the transform easier to apply, and faster to compute for realvalued input signals. In this paper, we present a simple change to the current definition of the DOST, and propose the Symmetric DOST (SDOST).

\section{REVIEW OF THE STOCKWELL TRANSFORM AND THE DOST}

The Stockwell transform, proposed in 1996 [1, 2, 3], gives a full time-frequency decomposition of a signal. Consider a one-dimensional function $h(t)$. The Stockwell transform of $h(t)$ is defined as the FT of the product between $h(t)$ and a Gaussian window function,

$$
S(\tau, f)=\int_{-\infty}^{\infty} h(t) \frac{|f|}{\sqrt{2 \pi}} e^{-\frac{(\tau-t)^{2} f^{2}}{2}} e^{-i 2 \pi f t} d t
$$

where $f$ is the frequency, and $t$ and $\tau$ are both time. In this way, the ST decomposes a signal into temporal $(\tau)$ and frequency $(f)$ components.

By the integral properties of the Gaussian function, the relation between $S(\tau, f)$ and $H(f)$ (the Fourier transform of $h(t))$ is

$$
\int_{-\infty}^{\infty} S(\tau, f) d \tau=H(f)
$$

That is, the accumulation of the Stockwell decomposition over time yields the Fourier coefficients of the signal, highlighting a special feature of the Stockwell transform. Hence, the original function $h(t)$ can be recovered by calculating the inverse Fourier transform of $H(f)$. 
Using the equivalent frequency domain definition of the Stockwell transform, the discrete Stockwell transform (DST) [1] can be written

$$
S\left[j T, \frac{n}{N T}\right]=\sum_{m=0}^{N-1} H\left[\frac{m+n}{N T}\right] e^{-2 \pi^{2} m^{2} / n^{2}} e^{i 2 \pi m j / N},
$$

for $n \neq 0$, and for the $n=0$ voice,

$$
S[j T, 0]=\frac{1}{N} \sum_{m=0}^{N-1} h[m T],
$$

which is analogous to the DC value of the FT.

The Stockwell transform has been applied in many fields such as geography [4, 5], engineering [6], and medical imaging for human functional brain mapping [7].

From (1), it is obvious that the Stockwell transform is an overcomplete representation. For a signal of length $N$, there are $N^{2}$ DST coefficients, but each one takes $O(N)$ to compute, suggesting that computing all $N^{2}$ coefficients of the DST has computational complexity of $O\left(N^{3}\right)$. That makes the Stockwell transform expensive to be applied to higher-dimensional cases. A more efficient basis is needed to pursue this time-frequency decomposition.

The DOST is a pared-down version of the fully redundant ST [8]. Since lower frequencies have longer periods, it stands to reason that lower frequencies can cope with lower sampling rates. Hence, the DOST subsamples the low frequencies. Similarly, spans of high frequencies have higher sample rates.

The DOST basis vectors are defined as

$$
S(k T)_{[v, \beta, \tau]}=\frac{1}{\sqrt{\beta}} \sum_{f=v-\beta / 2}^{v+\beta / 2-1} \exp \left(i 2 \pi \frac{k}{N} f\right) \exp \left(-i 2 \pi \frac{\tau}{\beta} f\right) \exp (-i \pi \tau),
$$

for $k=0, \cdots, N-1$, which can be summed analytically to

$$
S(k T)_{[v, \beta, \tau]}=i e^{-i \pi \tau} \frac{e^{-i 2 \alpha(v-\beta / 2-1 / 2)}-e^{-i 2 \alpha(v+\beta / 2-1 / 2)}}{2 \sqrt{\beta} \sin \alpha},
$$

where $\alpha=\pi(k / N-\tau / \beta)$ is the center of the temporal window and $v$ indicates the center of each frequency band voice with a bandwidth of $\beta$. To make the family of basis vectors in (6) orthonormal, the parameters, $\beta, v$ and $\tau$ have to be chosen suitably [8]. Thus a signal of length $N$ can be represented by the DOST with $N$ complex coefficients.

\section{SYMMETRIC DOST}

Unlike the FFT, the DOST coefficients of a real-valued input signal are not conjugate symmetric. This means that in real applications we have to calculate the positive frequency coefficients and the negative part as well.

To make the idea more clear, Fig. 1 shows how the parameters $v, \beta \& \tau$ partition the time-frequency domain. The partition corresponding to the DOST is shown in Fig. 1(a). Note that we are proposing a slightly different parameterization than in [8], using non-integer values for $v$.

We discovered that we can achieve conjugate symmetry by modifying the DOST basis slightly, still maintaining its orthogonality. We call this modified DOST the symmetric DOST (SDOST) in this paper. For real-valued signals, half of the computation can be avoided if we use the symmetric DOST.

If we impose the conjugate symmetry requirement on our DOST coefficients,

$$
s_{[v, \beta, \tau]}=\left(s_{[-v, \beta, \tau]}\right)^{*},
$$

then the basis functions for the SDOST are

$$
S(k T)_{[v, \beta, \tau]}=i e^{-i \pi \tau} \frac{e^{-i 2 \alpha(v-\beta / 2)}-e^{-i 2 \alpha(v+\beta / 2)}}{2 \sqrt{\beta} \sin \alpha},
$$

where $\alpha=\pi(k / N-\tau / \beta)$ is the center of the temporal window.

Equivalently, this change of definition amounts to shifting the time-frequency partitions by $1 / 2$ sample along the frequency axis. To implement this alternative, we replace $v$ by $v+1 / 2$ and use the same definition of the DOST as 


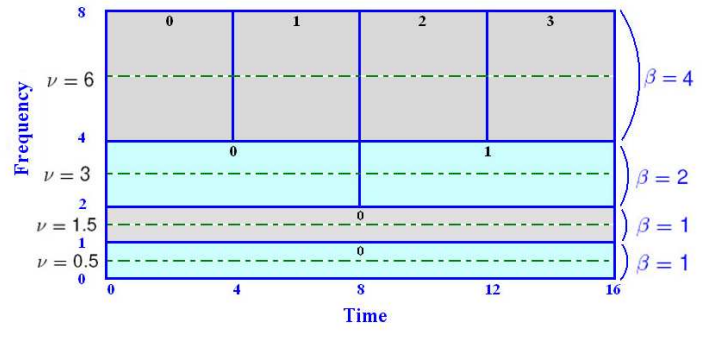

(a) Unified DOST.

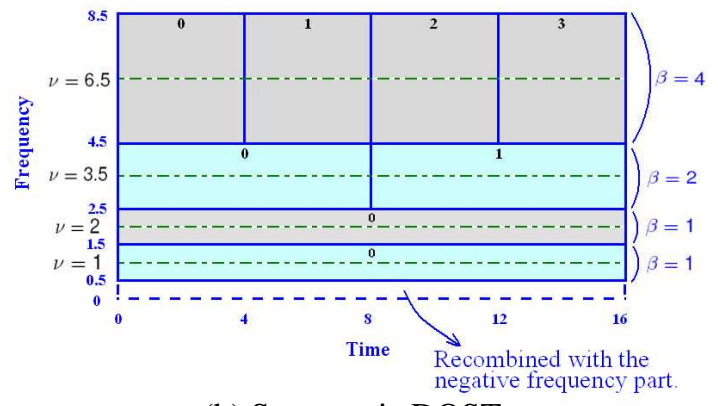

(b) Symmetric DOST

FIGURE 1. Partition diagram of the time-frequency domain. Each solid block corresponds to one DOST coefficient. The arrows in (a) show where we have modified the DOST by relaxing the integer constraint for $v$. In (b), the coefficients for the SDOST are shifted along the frequency axis by $\frac{1}{2}$.

(6). We have verified that the orthonormal property is held for the basis vector of the symmetric DOST. The partition over the time-frequency domain of SDOST is shown in Fig. 1(b).

We find that, by taking advantage of the FFT, the DOST coefficients can be calculated faster than by direct calculation (normally ten times faster). The strategy for this speedup is showed in Fig. 2(a). We also find that the SDOST can be calculated in a similar way (Fig. 2(b)) by multiplying the signal by a phase ramp. Note that the system matrix is slightly different for SDOST than for DOST. However, this transformation matrix is block-diagonal in both cases. Despite the fact that the SDOST corresponds to a 1/2-sample shift along the frequency axis, there is no loss of information to resampling because the shift is implemented (either implicitly or explicitly) using the Fourier shift theorem.

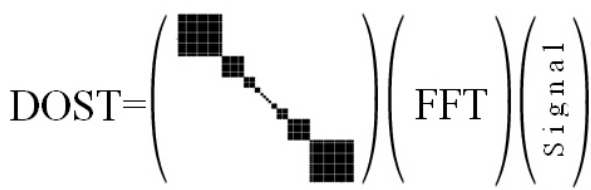

(a) DOST.

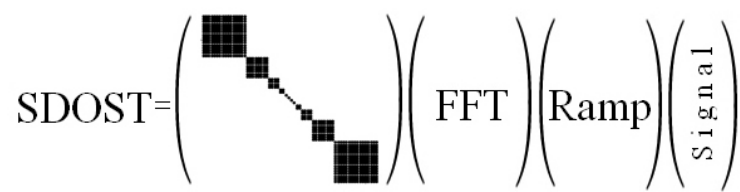

(b) SDOST

FIGURE 2. Calculation strategies of the DOST and the SDOST. The SDOST is equivalent to the shifted version of the DOST with a different transfer matrix.

\section{AN APPLICATION USING THE SYMMETRIC DOST}

We designed an experiment to test our method on a common signal-processing task, denoising. We constructed a signal by combining sine waves. The signal starts with a sinusoid with amplitude 1 and a high frequency $f_{\text {hi }}$, followed by a period of low frequency $f_{\text {lo }}$, and ending with the same high frequency $f_{\text {hi }}$. During the low frequency phase, we added high frequency noise with frequency $f_{\text {hi }}$. Our goal was to remove the high frequency noise without disturbing the high frequency parts at the beginning and end.

We used filters on the FFT, the DOST and the SDOST coefficients to try to remove the noise. From Fig. 3, we can see that the FFT has failed because the frequency content of the noise is similar to that of part of our desired signal. Due to the temporally localized nature of the frequency information, the DOST and the SDOST have both succeeded by returning almost the same denoised signal.

If we compare the denoised signal from the DOST to the SDOST more closely, the amplitude of the signal denoised by DOST is diminished to 0.955 , while the corresponding amplitude for SDOST is 0.968 . Why? Manipulation of the DOST coefficients during the filtering stage causes the reconstructed signal to no longer be real-valued; some of the energy of the original signal has been dispersed to the imaginary part of the signal. It is not clear how one would 
implement a filter on the DOST coefficients so that the reconstructed signal was real-valued. However, for the SDOST, we can assign the coefficients to zero symmetrically and get a real-valued signal after the reconstruction.

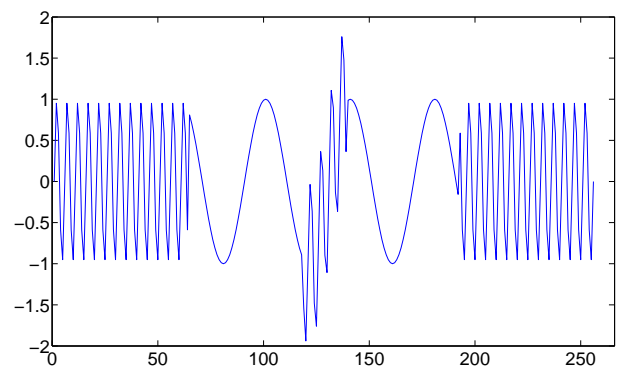

(a) Original signal.

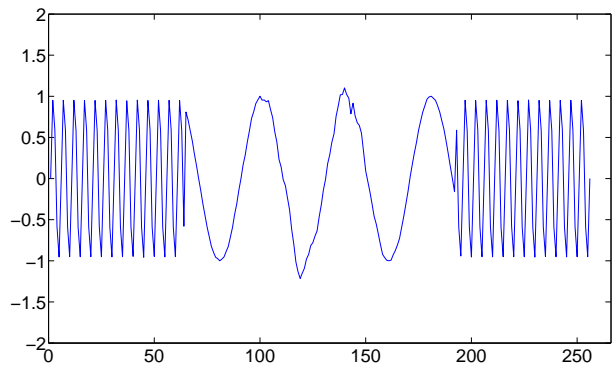

(c) DOST denoised signal.

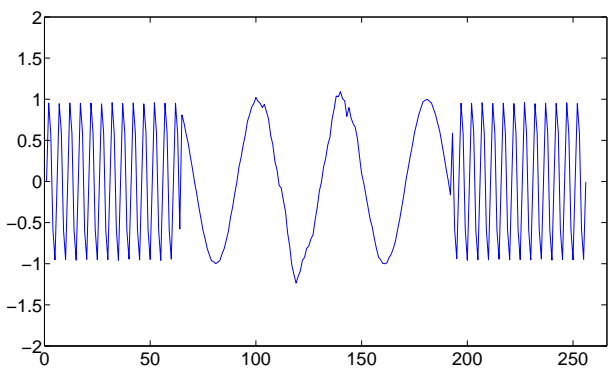

(b) SDOST denoised signal.

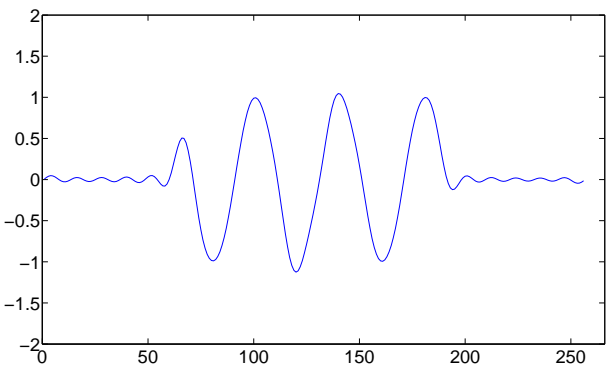

(d) FFT denoised signal.

FIGURE 3. Original signal and different versions of denoised signal.

\section{FURTHER WORK}

To compute the SDOST of a real-valued signal, we need to compute only half of the coefficients because the other half can be derived very easily from the symmetry property. Moreover, the DOST coefficients can be computed as a sparse linear mapping on the FFT of the data. While the computational complexity with these improvements is still $O\left(N^{2}\right)$, they may speedup computation of the DOST to a point where it is feasible in many more genres of application.

\section{REFERENCES}

1. Stockwell, R. G., Mansinha, L., and Lowe, R. P., "Localization of complex spectrum: the S transform," IEEE Transactions on Signal Processing 144, 998-1001 (April 1996).

2. Mansinha, L., Stockwell, R. G., Lowe, R., Eramian, M., and Schincariol, R. A., "Local S-spectrum analysis of 1-D and 2-D data," Phys. Earth Planet. Inter. 103, 3291 C336 (November 1997).

3. Eramian, M., Schincariol, R. A., Stockwell, R. G., Lowe, R. P., and Mansinha, L., "Review of applications of 1D and 2D S-transforms," Proc. SPIE 3078, 558-568 (April 1997).

4. Merzlyakov, E. G., Portnyagin, Y. I., Jacobi, C., Mitchell, N. J., Muller, H. G., Manson, A. H., Fachrutdinova, A. N., Singer, W., and Hoffmann, P., "On the longitudinal structure of the transient day-to-day variation of the semidiurnal tide in the mid-latitude lower thermosphere-i. Winter season," Ann. Geophys. 19, 545-562 (2001).

5. Oldenborger, G. A., Schincariol, R. A., and Mansinha, L., "Space-local spectral texture segmentation applied to characterizing the heterogeneity of hydraulic conductivity," Water Resouces Research 38(8), 1154-1157 (2002).

6. Dash, P., Panigrahi, B., and Panda, G., "Power quality analysis using S-transform," IEEE Transactions on Power Dilivery 18 (April 2003).

7. Andino, S., Menendez, R., Lantz, C., Blank, O., Michel, C., and Landis, T., "Non-stationary distributed source approximation: An alternative to improve localization procedures," Human Brain Mapping 14, 81-95 (July 2001). 
8. Stockwell, R. G., "A basis for efficient representation of the S-transform,” Digital Signal Processing 17, $371-393$ (January 2007). 